\title{
X-ray Fresnel diffractometry for ultralow emittance diagnostics of next generation synchrotron light sources
}

\author{
Mitsuhiro Masaki, Shiro Takano, Masaru Takao, and Yoshito Shimosaki \\ Japan Synchrotron Radiation Research Institute (JASRI/SPring-8), \\ 1-1-1 Kouto, Sayo, Hyogo 679-5198, Japan \\ (Received 5 June 2014; published 20 April 2015)
}

\begin{abstract}
A novel technique using single-slit x-ray Fresnel diffraction has been developed to resolve $\mu \mathrm{m}$-order electron beam sizes at the insertion devices (IDs) of photon beam lines. The new technique is promising for diagnostics of next-generation light sources, where a tuning of ultralow emittance at insertion devices is essentially important to ensure the absence of the degradation of brilliance and the transverse coherence of radiation at beam lines due to distortion of lattice functions. The validity of the method was experimentally studied at SPring-8, and the achievable resolution is discussed.
\end{abstract}

DOI: 10.1103/PhysRevSTAB.18.042802

PACS numbers: 29.20.db, 07.85.Qe, 41.85.Qg

\section{INTRODUCTION}

Electron storage ring light sources have provided high brightness photon beams at a repetition rate of the order of $\mathrm{MHz}$ in the spectral range from infrared to hard $\mathrm{x}$ rays. In recent years, ring-based next generation light sources have been extensively and intensively discussed, aiming to drastically boost the average brilliance and the transverse coherence by orders of magnitude compared with existing storage rings. One of the candidates is the so-called diffraction limited storage ring (DLSR) [1], which has an ultralow emittance of $100 \mathrm{pm}$ rad or less and $\mu \mathrm{m}$-order source sizes at the insertion devices (IDs) of photon beam lines. However, unwanted local distortions of betatron and dispersion functions at any point in a DLSR may exist due to inevitable magnetic field errors originating from misalignment and fabrication errors of strong quadrupole and sextupole magnets. Furthermore, a simple model of global betatron coupling [2,3] driven by a single differential resonance will no longer be applicable to a DLSR, and local betatron coupling [4] by effects of multiresonance lines will result in a position-dependent emittance coupling ratio along the ring. Therefore, to maximize the performance of the DLSR, it will be essentially important in terms of lattice optimization to measure the beam sizes at the source points of all IDs of the beam lines.

So far, various techniques have been developed to measure the $\mu \mathrm{m}$-order vertical beam sizes of the recent medium-size third-generation light sources, which have already obtained ultralow vertical emittance below a few pm rad by elaborate betatron coupling correction $[5,6]$. As

"masaki@spring8.or.jp

Published by the American Physical Society under the terms of the Creative Commons Attribution 3.0 License. Further distribution of this work must maintain attribution to the author $(s)$ and the published article's title, journal citation, and DOI. examples, for the Swiss Light Source, a small vertical beam size of $3.6 \mu \mathrm{m}$ has been measured [7] by the $\pi$-polarization imaging method [8], and for the Australian synchrotron storage ring, a small vertical emittance of $2.6 \mathrm{pm}$ rad has been evaluated by measuring radiation spectra from a vertical undulator [9], which can resolve a vertical emittance of the order of pm rad [10]. Recently, using the vertical undulator, even a vertical emittance of $0.9 \mathrm{pm}$ rad has been successfully observed [11]. X-ray pinhole cameras (XPCs) with a resolution of less than $10 \mu \mathrm{m}$ are also widely used, e.g., [12,13]. An X-ray imaging method using Fresnel zone plates (FZPs) [14,15], an interferometric technique [16,17], and a fluctuation analysis of incoherent synchrotron radiation [18] are also capable of resolving sub-ten $\mu \mathrm{m}$ beam sizes. However, these methods are not necessarily as readily applicable as they are to emittance diagnostics at all the ID source points of the beam lines in the DLSR. The $\pi$-polarization method and imaging by FZP require a bending magnet source, and vertical undulators are not universal as IDs. The interferometric technique and the fluctuation method use visible light for measurements. The addition of size-fixed $x$-ray pinholes specific for source emittance diagnostics to the user beam lines, which limits the apertures of the beam lines, would not be pragmatic. The pinhole image technique using a movable slit of the beam line requires an absolute accuracy of the narrow slit width of several tens of micrometer to evaluate a point spread function (PSF). Therefore, the development of a new emittance diagnostics technique universally applicable to all the ID beam lines is necessary to ensure that the proposed DLSRs can be used as real working light sources.

\section{THEORETICAL EXPLANATION}

We have developed a novel emittance diagnostic method based on X-ray Fresnel diffractometry (XFD). XFD observes a double-lobed diffraction pattern that emerges by optimizing a single slit width $A$ under given conditions 
of distance $L$ from a source point to the slit, distance $R$ from the slit to an observation point, and the observing wavelength $\lambda$, as shown in Fig. 1. The depth of the median dip in the double-lobed pattern correlates with the light source size; i.e., the dip becomes shallow with the increase in source size. The only requirement for light sources is that the radiation should be a spherical wave with a flux distribution wider than the slit width. Therefore, XFD is applicable to both bending magnet sources and most types of ID sources.

Assuming the spherical wave approximation in a onedimensional case for simplification, a PSF at the observation screen is expressed by the following Fresnel integral using the parabolic approximation of the spherical phase:

$$
\begin{aligned}
I\left(y, y_{e}\right) \propto & \propto \int_{-A / 2}^{A / 2} \sqrt{I_{s}\left(y_{s}-y_{e}\right)} \exp \\
& \times\left.\left[i \frac{\pi}{\lambda}\left\{\frac{1}{L}+\frac{1}{R}\right\}\left\{\left(y_{s}-y_{e}\right)-\frac{L\left(y-y_{e}\right)}{L+R}\right\}^{2}\right] d y_{s}\right|^{2},
\end{aligned}
$$

where $I_{s}\left(y_{s}-y_{e}\right)$ is the flux intensity distribution of radiation at the slit, $y_{e}$ is an electron position at the source point, and $y_{s}$ and $y$ are coordinates on the slit and the screen, respectively. Here, we let the electron position $y_{e}=0$. Assuming a constant flux distribution at the slit, the slit width $A$ required to create a double-lobed PSF with the deepest median dip is expressed as follows:

$$
A \approx \sqrt{7 \lambda \frac{L R}{L+R}}
$$

This formula is derived from the condition of destructive interference of the light contributing to the center $(y=0)$ of the PSF, which minimizes the intensity $I(0)$ in Eq. (1). A numerical calculation shows that $I(0)$ is minimized when the quadratic phase variation from the slit center to both edges $(A / 2$ and $-A / 2)$ is about $7 \pi / 4$ rather than $2 \pi$. The coefficient given as 7 in Eq. (2) results from a phase variation of $7 \pi / 4$. The distance between two lobe peaks, i.e., the pitch $P$, is given by the following formula derived from a condition of constructive interference of the light:

$$
P=2 \lambda \frac{R}{A} \approx 2 \sqrt{\frac{\lambda}{7} \frac{R(L+R)}{L}} .
$$

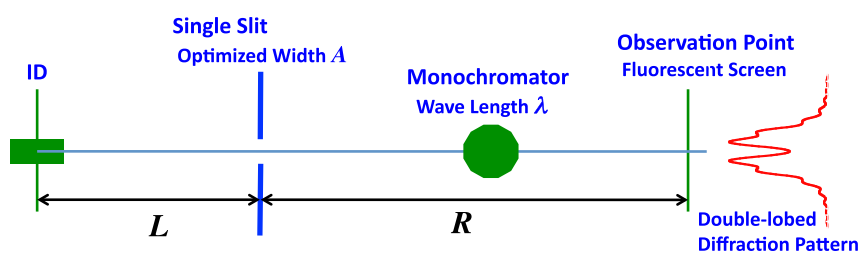

FIG. 1. A schematic for the source size measurement of an ID using XFD.
The XFD scheme requires a monochromatic $x$ ray. According to a numerical analysis, when the peak-to-peak photon bandwidth is narrower than $\Delta \lambda / \lambda \approx 2 \%$, the PSF modification due to the finite bandwidth is negligibly small. Silicon (111) is widely used as a monochromator crystal, and generates a photon bandwidth of $10^{-4}$, which is the sufficiently narrower than $2 \%$.

The undulator and wiggler radiations have the light properties required by XFD. The wavefront is well approximated by a spherical wave with a quadratic phase distribution around the longitudinal light axis [19] where the slit is placed. Furthermore, the flux of the on-axis resonant wavelength comprises a spatial distribution with a flat top that is wider than a slit width of several tens of micrometer [19]. When the slit width $A$ is expanded in comparison to the condition given by Eq. (2), different Fresnel diffraction patterns emerge with more complicated interference fringes, which have been used for measuring vertical beam sizes at undulator source points [20]. However, a wider slit can create non-negligible effects of angular divergence of the electron beam on the observed diffraction pattern due to contributions of the outer side of the flat top region where $I_{s}\left(y_{s}-y_{e}\right)$ is not constant. Therefore, we hold that the simpler double-lobed pattern obtained with the narrower slit is preferable.

\section{NUMERICAL EXAMPLE FOR SOURCE SIZE MEASUREMENTS AT DLSR}

We simulated the source size measurement using XFD at a DLSR beam line, assuming a distance of $L=25 \mathrm{~m}$ from the source to the slit, $R=25 \mathrm{~m}$ from the slit to the screen, and an x-ray energy of $40 \mathrm{keV}$. From Eq. (2), the optimized slit width under these conditions is $52 \mu \mathrm{m}$. Figure 2 shows the calculated PSF assuming a constant $I_{s}\left(y_{s}-y_{e}\right)$ and its convolution with Gaussian-distributed sources. The peakto-valley intensity ratio $I_{v} / I_{p}$ of the median dip exhibits a high sensitivity to $\mu \mathrm{m}$-order changes in the root mean square (rms) source size of less than $10 \mu \mathrm{m}$, corresponding

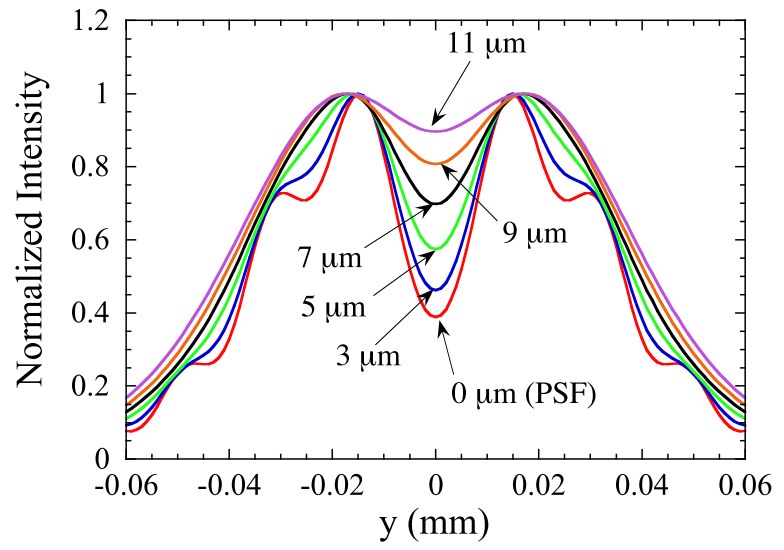

FIG. 2. The calculated PSF and its convolution with Gaussiandistributed sources having sizes from 3 to $11 \mu \mathrm{m}$ (rms), assuming vertical source size measurements using XFD with an $\mathrm{x}$-ray energy of $40 \mathrm{keV}$ at DLSR beam lines. 


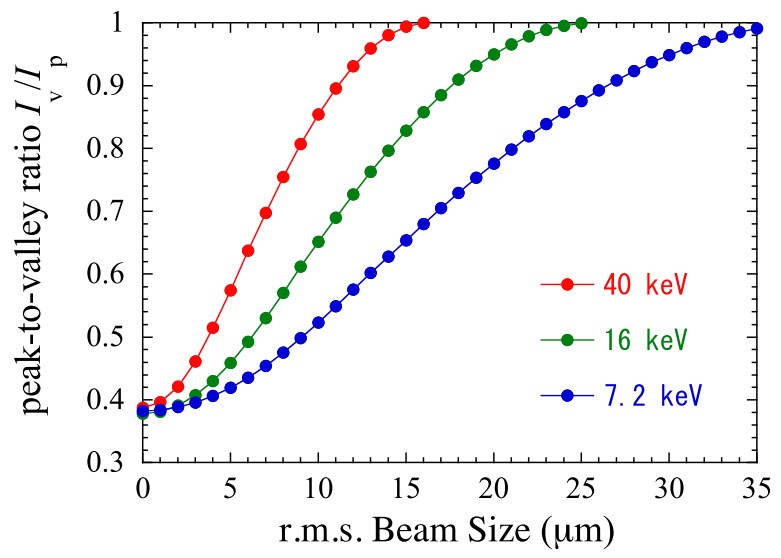

FIG. 3. Sensitivity curves calculated at observing x-ray energies of 40, 16, and $7.2 \mathrm{keV}$.

to vertical electron beam sizes at the ID source points, where a vertical emittance from 10 to $20 \mathrm{pm}$ rad and a vertical betatron function of several meters are assumed. Sensitivity curves, given by the ratio $I_{v} / I_{p}$ as a function of the source size, are shown in Fig. 3 for x-ray energies of 40, 16 , and $7.2 \mathrm{keV}$, where the slit width is optimized for each $\mathrm{x}$-ray energy. The observing $\mathrm{x}$-ray energy can be tuned by a beam line monochromator depending on the range of the measuring source sizes. XFD using a photon energy of $7.2 \mathrm{keV}$ is also applicable to the measurement of horizontal source sizes of about $20 \mu \mathrm{m}$ corresponding to an ultralow horizontal emittance of about $100 \mathrm{pm}$ rad of a DLSR.

\section{EXPERIMENTAL STUDY}

\section{A. Demonstration of XFD}

We demonstrated source size measurement using XFD at the SPring-8 diagnostics beam line (BL05SS) [21] with a planar undulator (ID05) [22]. The slit to form the diffraction pattern is located at a distance of $L=26.8 \mathrm{~m}$ from the source point, and the observation point at a distance of $R=65.4 \mathrm{~m}$ from the slit. The double-lobed patterns were observed using a high-resolution $\mathrm{x}$-ray imaging system (HAMAMATSU), which comprises a P43 fluorescent screen, imaging optics, and a CCD camera. The resolution of the imaging system was calibrated by the sharpness of the observed edge of a $0.5-\mathrm{mm}$ diameter stainless steel wire placed in front of the imaging system. The calibrated resolution of $16.6 \mu \mathrm{m}$ (rms) is scaled to $6.8 \mu \mathrm{m}$ (rms) at the source point owing to division by the magnification factor $R / L$. The exposure time of the CCD camera was set at a minimum of $1 \mathrm{~ms}$ to reduce the influence of the vibration of the cryogenically cooled monochromator crystals at a distance of $r=22.7 \mathrm{~m}$ upstream of the observation point. The angular fluctuation of the monochromatic x-ray beam caused by the vibration during the exposure was evaluated to be typically $0.3 \mu \mathrm{rad}$ (rms) by measuring the $\mathrm{x}$-ray beam position every $0.1 \mathrm{~ms}$ using an existing turn-by-turn profile monitor [23] with a fast CCD camera. It corresponds to an equivalent source oscillation of $3 \mu \mathrm{m}$ (rms) obtained by dividing the position fluctuation $(0.3 r \mu \mathrm{m})$ at the observation point by the magnification factor $R / L$. An X-ray energy of $7.2 \mathrm{keV}$ for the first harmonic radiation was selected by the monochromator. The x-ray energy was fine-tuned to $7.167 \mathrm{keV}$ within the spectrum band width of the harmonic radiation to maximize the flat top width of the vertical flux distribution observed with the slit fully opened. Since the optimized slit width given by Eq. (2) assumes a constant flux, the slit must be positioned in the flat top region. Owing to the maximization of the flat top width, we can obtain a wider acceptable range of slit positioning. Furthermore, if the flat top part of the flux distribution deviates from the slit due to the drift and fluctuation of the light axis, an unwanted asymmetric diffraction pattern can emerge. The wider flat top can broaden the tolerable range of the deviation. The experimentally adjusted vertical slit width found to provide the deepest median dip in the observed pattern was consistent with the theoretical width of $150 \mu \mathrm{m}$ from Eq. (2). A horizontal slit of $200 \mu \mathrm{m}$ was also empirically adjusted to produce the deepest dip for minimizing the peak-to-valley contrast degradation of the vertical PSF due to off-axis radiations with narrower or no flat top deriving from the horizontal emittance (2.4 nm rad) of the current SPring-8 storage ring. Here, note that in a DLSR with an ultralow horizontal emittance, the effect of the off-axis radiations resulting in PSF blurring will be negligibly small.

To confirm that XFD has a sensitivity to changes in the ID source size, the double-lobed diffraction patterns were observed for various vertical emittance conditions. For this purpose, we turned on and off the skew quadrupole magnets for the betatron coupling correction, and moved the operation point of the ring toward a differential coupling resonance line by changing the horizontal betatron tunes $\nu_{x}$ from 41.133 to 41.361 with the vertical betatron tune $\nu_{y}$ fixed to 19.34. Figure 4 shows four examples of the observed Fresnel diffraction images. The double-lobed structures are clearly observed in the vertical direction; however, they are smeared in the horizontal direction due to the large horizontal emittance. The normalized vertical lineprojected profiles of the four two-dimensional (2D) images are depicted in Fig. 5. The profiles (a) and (b) in Fig. 5 show
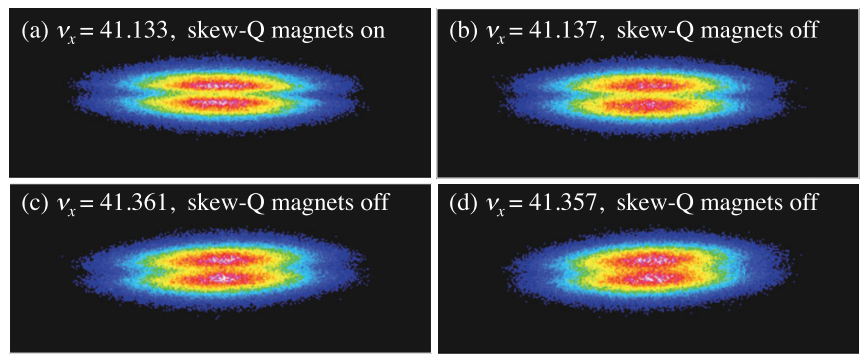

FIG. 4. Examples of the double-lobed diffraction images observed by changing betatron coupling conditions. 


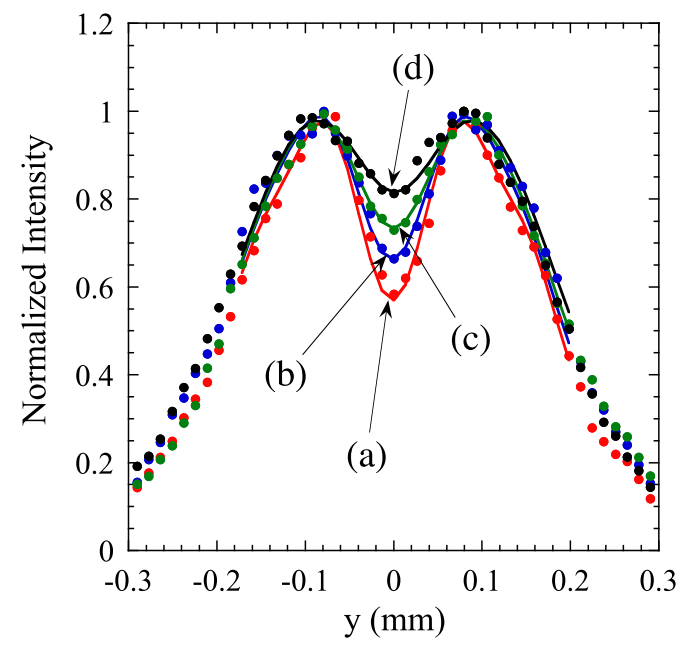

FIG. 5. Normalized vertical line-projected profiles (dots) of the four 2D images given in Fig. 4 and their fitted curves (solid lines) by Gaussian source distributions convolved with the PSF. The red, blue, green, and black colors correspond to (a), (b), (c), and (d) in Fig. 4, respectively.

the diffraction patterns with and without the coupling correction by the skew quadrupole magnets at the operation point where the effect of the coupling resonance line is negligible. The observed dip with the correction is clearly deeper than that without the correction. The diffraction patterns (b), (c), and (d) in Fig. 5 obtained with the skew quads switched-off, reflect the difference of the distance from the resonance line, i.e., the fractional part of $\left|\nu_{x}-\nu_{y}\right|$. The observed dip becomes less deeper with the growth of the betatron coupling as the operation point gets closer to the resonance line.

To evaluate the vertical beam sizes at ID05 from the observed vertical profiles, the vertical PSF of this experimental setup is needed. For a strict PSF calculation, we have to consider the effects of the horizontal emittance $\epsilon_{x}$ and the relative energy spread $\sigma_{E} / E$ of the electron beam. Each electron in the beam creates a different flux distribution depending on the off-axis and off-resonant conditions arising from $\epsilon_{x}$ and $\sigma_{E} / E$, which results in a different shape of the PSF. In the calculation of the PSF required to evaluate the vertical beam sizes from the measured double-lobed diffraction patterns, we have to average the shape of the PSF of each electron over the horizontal emittance and the energy spread. The calculation of Eq. (1) considering only the $I_{s}\left(y_{s}-y_{e}\right)$ for an on-axis and on-resonant single electron, which is shown in Fig. 6 by the blue curve, dose not lead to the accurate PSF. We calculated the vertical PSF strictly by folding the horizontal emittance $\epsilon_{x}=2.4 \mathrm{~nm}$ rad and the energy spread $\sigma_{E} / E=$ $0.11 \%$ of the SPring- 8 storage ring. A practical alternative to the strict PSF by the rigorous treatment described above is an effective PSF calculated by Eq. (1) using a flux distribution $I_{s}\left(y_{s}-y_{e}\right)$ measured experimentally with the

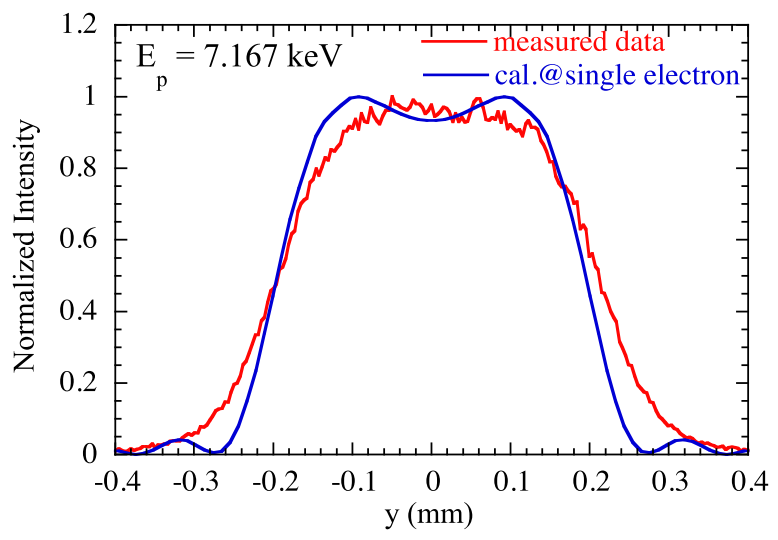

FIG. 6. The normalized vertical flux distributions at $7.167 \mathrm{keV}$ on the first harmonic of ID05. Blue and red lines show the calculation of radiation from a single electron with on-axis and on-resonant condition, and the experimental measurement with the vertical slit fully opened, respectively.

slit fully opened in the vertical, as shown by the red distribution in Fig. 6. The strict and effective PSFs are shown in Fig. 7. The two vertical PSFs create a systematic discrepancy of about $5 \%$ for $10 \mu \mathrm{m}$ (rms) in the evaluated beam sizes. The discrepancy is small and practically not so critical.

Using the strictly calculated PSF by folding $\epsilon_{x}$ and $\sigma_{E} / E$ (Fig. 7), the vertical beam sizes were evaluated by fitting Gaussian source distributions convolved with the PSF to the measured line-projected profiles in Fig. 5. The fitted function $f(y)$ is expressed as

$$
f(y)=C \int_{-\infty}^{\infty} I\left(y, y_{e}\right) \exp \left[-\frac{\left(y_{e}-y_{0}\right)^{2}}{2\left(\sigma_{y, e}^{2}+\sigma_{r e s}^{2}\right)}\right] d y_{e},
$$

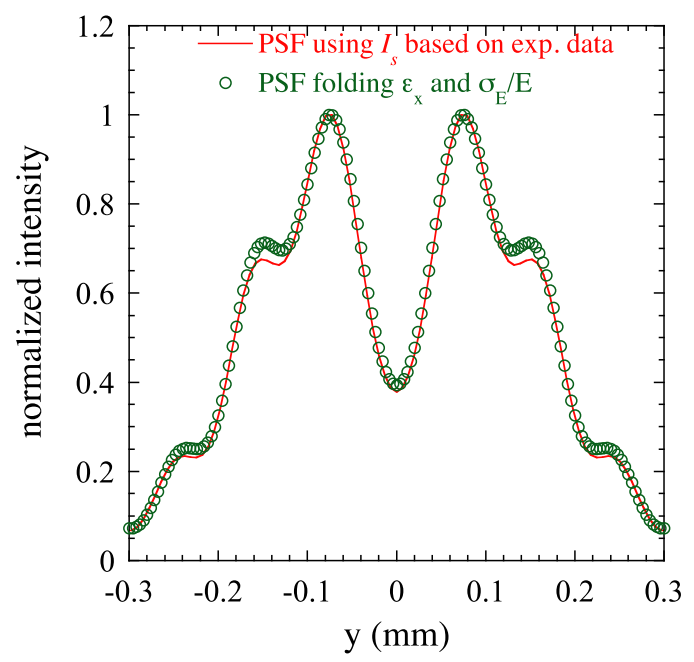

FIG. 7. Two calculated vertical PSFs. The red line shows the effective PSF using the measured flux distribution as $I_{s}\left(y_{s}-y_{e}\right)$. Green circles show the strict PSF folding the contributions of the off-axis and off-resonant radiations from $\epsilon_{x}=2.4 \mathrm{~nm} \mathrm{rad}$ and $\sigma_{E} / E=0.11 \%$. 
where the free parameters are the normalization factor $C$, center position $y_{0}$, and vertical electron beam size $\sigma_{y, e}$. The imaging system resolution $\sigma_{r e s}$ is $6.8 \mu \mathrm{m}$ (rms), as mentioned above. The smallest among the best-fitted beam sizes is $7.8 \mu \mathrm{m}$ (rms) for the deepest diffraction pattern shown by (a) in Fig. 5. Therefore, a light source size at an ID smaller than $10 \mu \mathrm{m}$ was successfully resolved by XFD.

\section{B. Experimental validation of XFD}

To experimentally verify the validity of XFD, we measured the vertical beam sizes at the ID05 source point while changing the vertical emittance systematically. The vertical beam size $\sigma_{y}$ is expressed by the following formula:

$$
\sigma_{y}=\sqrt{\beta_{y}\left(\epsilon_{y, c}+\epsilon_{y, \mathrm{rad}}\right)+\left[\eta_{y, s} \frac{\sigma_{E}}{E}\right]^{2}},
$$

where $\beta_{y}$ and $\eta_{y, s}$ are the betatron function and the dispersion at the source point in the vertical direction, respectively. The parameters $\epsilon_{y, c}$ and $\epsilon_{y, \text { rad }}$ are the projected vertical emittances arising from the $\mathrm{X}-\mathrm{Y}$ betatron coupling and the radiation excitation induced by the vertical dispersions $\eta_{y}$ along the ring [24], respectively. To change $\epsilon_{y, \text { rad }}$, the vertical dispersions were systematically induced by exciting the skew quadrupole magnets under the constraint of the constant driving term of the linear coupling resonance, i.e., keeping the parameter $\epsilon_{y, c}$ constant [25]. Figure 8 shows the vertical dispersion distributions measured using the beam position monitors (BPMs) located along the ring.

For each set of the dispersion distribution, the vertical beam sizes at the ID05 source point were measured by XFD. For comparison, we also simultaneously measured vertical beam sizes using two other existing beam size monitors with separate bending magnet sources (38B2 and 47B2): the $\mathrm{x}$-ray beam imager (XBI) [15] by a Fresnel zone plate at $38 \mathrm{~B} 2$ and the 2D-interferometric technique (2D-Int) [17] by a diffraction mask with four circular apertures instead of a double slit at 47B2. Figure 9 shows the measured vertical beam sizes at the three source points. The error bars showing the statistical errors $(1 \sigma)$ are

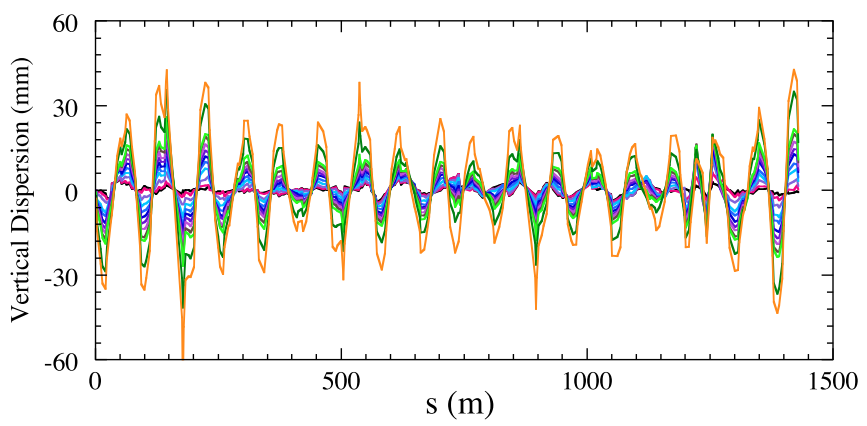

FIG. 8. Vertical dispersion distributions induced by the skew quadrupole magnets, measured by the BPMs.

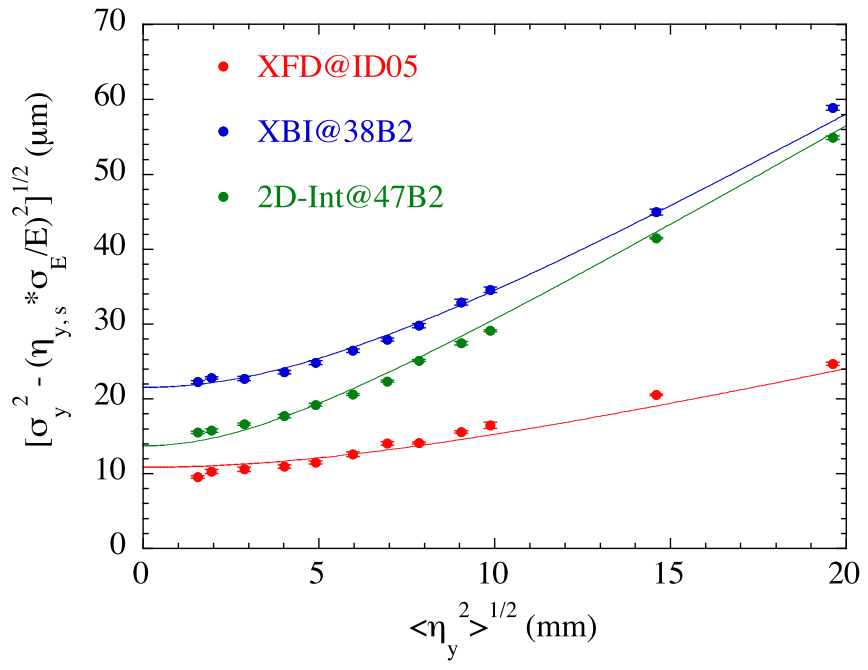

FIG. 9. Results of simultaneous measurements using XFD, $\mathrm{XBI}$, and 2D-Int at three different source points: ID05, 38B2, and 47B2. Solid lines show the best-fitted curves to each data set.

comparable with the symbol size. In the XFD measurement, the strict PSF folding $\epsilon_{x}$ and $\sigma_{E} / E$ shown in Fig. 7 was used to evaluate the beam sizes. The abscissa of Fig. 9 represents the rms values $\sqrt{\left\langle\eta_{y}^{2}\right\rangle}$ of the vertical dispersion distributions measured by the BPMs. The ordinate only indicates the contribution of the vertical emittance, $\sqrt{\sigma_{y}^{2}-\left(\eta_{y, s} \frac{\sigma_{E}}{E}\right)^{2}}$, subtracting the contribution of $\sigma_{E} / E=$ $0.11 \%$ from the measured vertical beam sizes, where the vertical dispersions at each source point were interpolated using transfer matrices from the measured dispersions at two BPMs adjacent to the sources. The following theoretical model function is fitted to these experimental data sets for the three source points:

$$
f(x)=\sqrt{\beta_{y}\left(\epsilon_{y, c}+D x^{2}\right)},
$$

where $x=\sqrt{\left\langle\eta_{y}^{2}\right\rangle}$. The vertical emittance $\epsilon_{y, \text { rad }}$ is approximately expressed by the second term $D\left\langle\eta_{y}^{2}\right\rangle$ [25] (see Fig. 11 in the Appendix). The parameter $D$ is independent of the source positions. The parameter $\epsilon_{y, c}$ can be different at the three source points. Therefore, we have four free parameters: a common parameter $D$ and $\epsilon_{y, c}$ for each source. The vertical betatron functions $\beta_{y}$ are fixed parameters of $4.88 \mathrm{~m}, 30.9 \mathrm{~m}$, and $31.9 \mathrm{~m}$ at the ID05, 38B2, and 47B2 sources, respectively, which are calculated values considering normal and skew quadruple magnetic field errors evaluated using a response matrix analysis [26]. The best-fitted results of the four parameters are listed in Table I.

The experimental results obtained by XFD, XBI, and 2D-Int were well fitted by the theoretical curves with the common coefficient $D$. To examine the validity of the obtained value of the $D$, we calculated the parameter $D$ 
TABLE I. The best-fitted results of the common coefficient $D$ and $\epsilon_{y, c}$ at the three source points: ID05, 38B2, and 47B2.

\begin{tabular}{lccc}
\hline \hline$D\left(\mathrm{pm} \mathrm{rad} / \mathrm{mm}^{2}\right)$ & \multicolumn{3}{c}{$\epsilon_{y, c}(\mathrm{pm} \mathrm{rad})$} \\
\hline & ID05 (XFD) & $38 \mathrm{~B} 2(\mathrm{XBI})$ & $47 \mathrm{~B} 2(2 \mathrm{D}-\mathrm{Int})$ \\
0.235 & 24.0 & 15.0 & 5.88 \\
\hline \hline
\end{tabular}

theoretically for the current SPring-8 storage ring. The value of $D$ experimentally determined (Table I) is in good agreement with the calculated value of $0.235 \mathrm{pm} \mathrm{rad} / \mathrm{mm}^{2}$ (see Appendix for details). We note that the deviations of the XFD results from the theoretical fitted curve are practically small, residing within $1.5 \mu \mathrm{m}$, nonetheless larger than a typical statistical error of $0.26 \mu \mathrm{m}(1 \sigma)$. We are planning to further investigate the possibility of residual systematic errors as a cause of the practically small but statistically significant deviations. Other possibilities, for example, the assumption of the preservation of the constant betatron coupling during the experiments would be examined.

To examine the different values of $\epsilon_{y, c}$ obtained for the three sources (Table I), we simulated the equilibrium vertical emittance at each source point under the condition of the minimum rms vertical dispersion $(1.56 \mathrm{~mm})$ corresponding to the leftmost data point in Fig. 9, where the vertical emittance is dominated by the betatron coupling. The theoretical vertical emittances (shown in Table II) were obtained by tracking 1000 particles using a six-dimensional symplectic tracking code with differential algebra (CETRA) [27], considering the magnetic field errors evaluated by the response matrix analysis as mentioned above. The corresponding experimental vertical emittances evaluated from the measured beam sizes (leftmost point in Fig. 9) are also given in Table II. The experimental results indicate a similar tendency with the theoretical simulation with respect to the relative magnitudes among the three sources, the maximum value being about three times larger than the minimum. We deduce that the differences of the vertical emittances among the three sources is owing to the influence of local betatron coupling [4] resulting in a position-dependent $\mathrm{X}-\mathrm{Y}$ emittance coupling ratio along the ring.

The ID source size measurements by XFD while systematically controlling the vertical emittance are consistent with the theoretical simulation. The validity of XFD has been experimentally verified, and successfully

TABLE II. Evaluated vertical emittances $\epsilon_{y}$ under the condition of the minimum rms vertical dispersion $(1.56 \mathrm{~mm})$.

\begin{tabular}{lccc}
\hline \hline Source & Monitor & Simulation (pm rad) & Experiment (pm rad) \\
\hline ID05 & XFD & 18.2 & $18.6 \pm 0.6$ \\
38B2 & XBI & 10.8 & $16.0 \pm 0.3$ \\
47B2 & 2D-Int & 7.48 & $7.52 \pm 0.13$ \\
\hline \hline
\end{tabular}

demonstrating that XFD is a well-workable method for emittance diagnostics of a ID source point.

\section{RESOLUTION OF XFD COMPARED WITH XPC}

The experiments conducted at SPring-8 have shown that XFD is sensitive to changes in the beam size at the ID source point. If a higher x-ray energy, such as $40 \mathrm{keV}$ shown in Fig. 2, is selected, XFD would enable the measurement of a source size of a few micrometers. Furthermore, the XFD has the potential to obtain higher sensitivity to source sizes of about $1 \mu \mathrm{m}$ (rms) by placing the slit at a shorter distance of several meters from a source point as with an XPC. In the usual XPC, the pinhole is placed as close to the source as possible to obtain high sensitivity with a resolution of several micrometers, and the magnification factor is greater than one $[12,13]$. For example, in the case of $L=3 \mathrm{~m}$ from the source to the pinhole (slit), $R=9 \mathrm{~m}$ from the pinhole (slit) to the screen, and an X-ray energy of $40 \mathrm{keV}$, we compare the resolution of XFD with that of an XPC. The optimized pinhole size of an XPC is given by the following formula based on calculation of the Fresnel diffraction [13]:
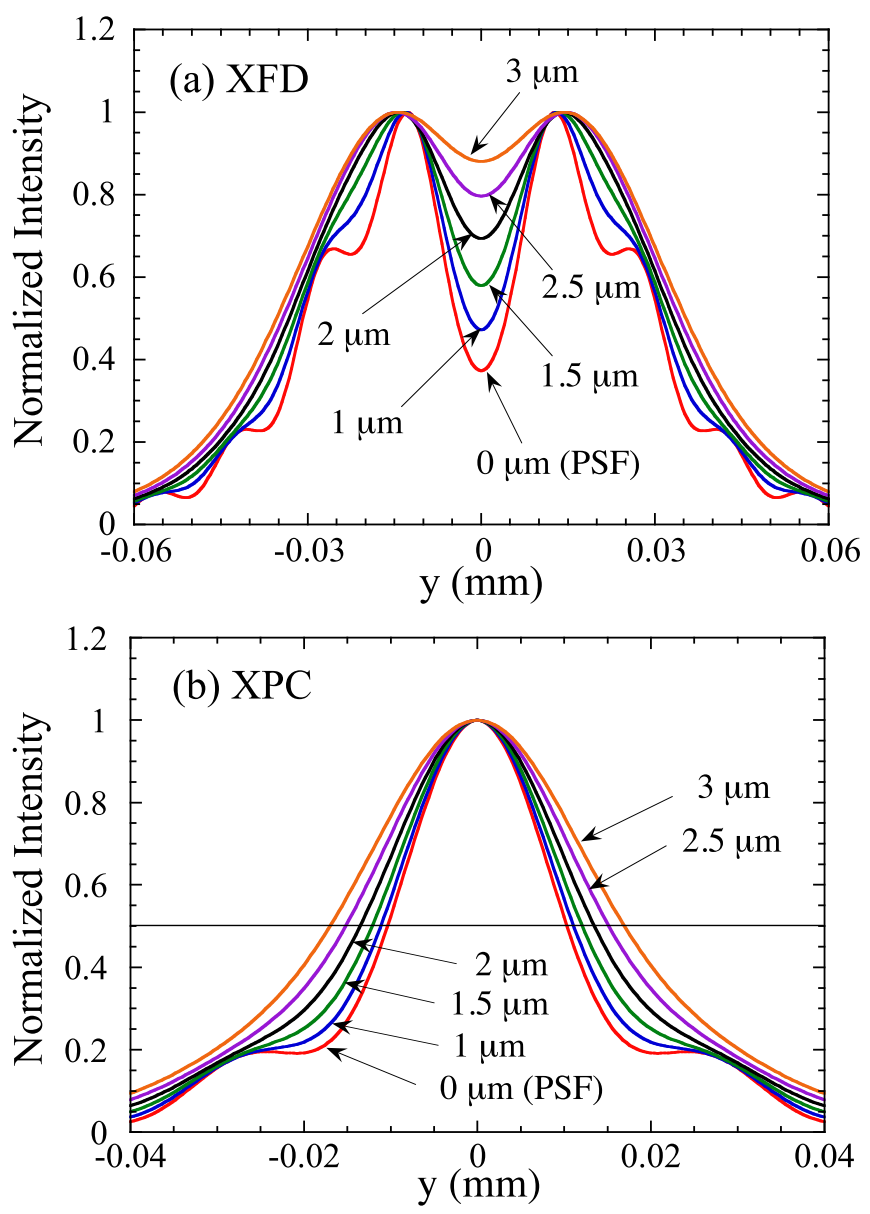

FIG. 10. PSFs and their convolutions with Gaussian-distributed sources of rms sizes from 1 to $3 \mu \mathrm{m}$ for (a) XFD with a slit width of $22 \mu \mathrm{m}$ and (b) XPC with a pinhole size of $13 \mu \mathrm{m}$. 


$$
A=\sqrt{\frac{8 \lambda}{\pi} \frac{L R}{L+R}}
$$

In both cases, the PSFs and their convolutions with Gaussian-distributed sources are calculated for the optimized slit and pinhole sizes of $22 \mu \mathrm{m}$ for XFD and $13 \mu \mathrm{m}$ for XPC. As shown in Fig. 10, when the source size is $1 \mu \mathrm{m}$ (rms), in the case of XFD, the bottom intensity of the dip increases by $26 \%$ compared with that in the case of the PSF. On the other hand, in XPC, the full width at half maximum (FWHM) of the convoluted pattern broadens by $8 \%$ compared with that in the PSF. The increase of the bottom intensity in XFD with the source size is more remarkable than the broadening of the FWHM width in XPC. Therefore, we can see that XFD has a higher sensitivity to changes in $\mu \mathrm{m}$-order source sizes than the case of XPC, and the example given in Fig. 10(a) indicates that XFD can resolve source sizes even less than $1 \mu \mathrm{m}$ (rms). Observation of the double-lobed pattern with a small pitch of about $30 \mu \mathrm{m}$ as shown in Fig. 10(a) requires an imaging with a high optical resolution. It will be possible by the use of a thin fluorescent screen of less than $10 \mu \mathrm{m}$ to avoid blurring on the screen and a visible light imaging optics with a magnification factor of 2-3.

\section{CONCLUSION}

We have newly developed x-ray Fresnel diffractometry (XFD) for ultralow emittance diagnostics at ID source points of photon beam lines. The new technique is available at typical beam lines equipped with a 4-jaw slit and a monochromator, and useful for optimizing storage ring lattice functions to maximize the brilliance and the transverse coherence at each photon beam line. Furthermore, XFD with an optimized aperture close to the source point has the potential to resolve beam sizes of less than $1 \mu \mathrm{m}$. $\mathrm{XFD}$ is a promising emittance diagnostic method to maximize the performance of ring-based next-generation light sources.

\section{ACKNOWLEDGMENTS}

We would like to thank K. Soutome, T. Nakamura, T. Watanabe, K. Tamura, and H. Ohkuma for their useful discussions and comments, and Nano-forensic Science Group of SPring-8 for their kindness in allowing us to use the high-resolution $\mathrm{x}$-ray imaging system for this experiment.

\section{APPENDIX: VERTICAL EMITTANCE INDUCED BY VERTICAL DISPERSION}

The vertical emittance arising from radiation excitation induced by vertical dispersion is expressed by the following formulas [28]:

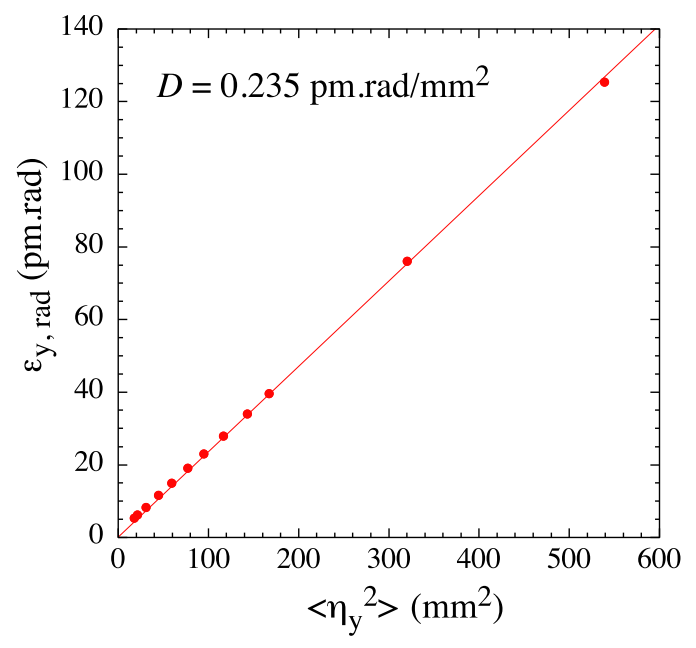

FIG. 11. The calculated vertical emittances arising from radiation excitation for the current SPring- 8 storage ring as a function of $\left\langle\eta_{y}^{2}\right\rangle$ using the dispersions evaluated at the BPM positions.

$$
\begin{aligned}
\epsilon_{y, \mathrm{rad}} & =C_{q} \gamma^{2} \frac{\oint\left(H_{y} / \rho^{3}\right) d s}{J_{y} \oint\left(1 / \rho^{2}\right) d s}, \\
H_{y} & =\frac{1}{\beta_{y}}\left[\eta_{y}{ }^{2}+\left(\beta_{y} \eta_{y}^{\prime}-\frac{1}{2} \beta_{y}^{\prime} \eta_{y}\right)^{2}\right],
\end{aligned}
$$

where $C_{q}=3.83 \times 10^{-13} \mathrm{~m}, \gamma$ is the Lorentz factor, $\rho$ is the dipole bending radius, and $J_{y} \approx 1$ is the vertical damping partition number. The vertical emittances $\epsilon_{y \text {,rad }}$ corresponding to each vertical dispersion distribution in Fig. 8 were calculated using Eqs. (A1) and (A2). The betatron function $\beta_{y}$, the dispersion $\eta_{y}$ in the vertical direction, and their derivatives $\beta_{y}^{\prime}$ and $\eta_{y}^{\prime}$ were calculated by considering the magnetic error fields and the excited skew quadrupole magnetic fields, as mentioned above. As shown in Fig. 11, the emittance is approximately proportional to the square of the rms of the dispersion distribution along the ring, where the rms values were obtained using the calculated dispersions at the BPM positions. We obtained the coefficient $D=0.235 \mathrm{pm} \mathrm{rad} / \mathrm{mm}^{2}$ using a linear fitting.

[1] R. Hettel and M. Borland, in Proceedings of the 25th Particle Accelerator Conference, PAC-2013, Pasadena, CA, 2013 (IEEE, New York, 2013), p. 19.

[2] M. Takao, Phys. Rev. ST Accel. Beams 9, 084002 (2006).

[3] M. Masaki, M. Takao, K. Soutome, and S. Takano, Phys. Rev. ST Accel. Beams 12, 024002 (2009).

[4] A. Franchi, L. Farvacque, J. Chavanne, F. Ewald, B. Nash, and K. Scheidt, Phys. Rev. ST Accel. Beams 14, 034002 (2011).

[5] M. Aiba, M. Böge, N. Milas, and A. Streun, Nucl. Instrum. Methods Phys. Res., Sect. A 694, 133 (2012).

[6] R. Dowd, M. Boland, G. LeBlanc, and Y.-R. E. Tan, Phys. Rev. ST Accel. Beams 14, 012804 (2011). 
[7] V. Schlott, M. Rohrer, A. Saa Hernandez, A. Streun, A. Andersson, J. Breunlin, and N. Milas, in Proceedings of the 2nd International Beam Instrumentation Conference 2013 (JACoW, Oxford, United Kingdom, 2013), p. 519.

[8] Å. Andersson, M. Böge, A. Lüdeke, V. Schlott, and A. Streun, Nucl. Instrum. Methods Phys. Res., Sect. A 591, 437 (2008).

[9] K. P. Wootton, M. J. Boland, R. Dowd, Y.-R.E. Tan, B. C. C. Cowie, Y. Papaphilippou, G. N. Taylor, and R. P. Rassool, Phys. Rev. Lett. 109, 194801 (2012).

[10] S. Takano, in Proceedings of the Workshop on Precise Measurements of Electron Beam Emittance, KEK Proceedings, Vol. 97-20 (KEK, Tsukuba, Japan, 1998), p. 17.

[11] K. P. Wootton, M. J. Boland, and R. P. Rassool, Phys. Rev. ST Accel. Beams 17, 112802 (2014).

[12] M.-A. Tordeux, L. Cassinari, O. Chubar, J.-C. Denard, D. Pédeau, and B. Pottin, in Proceedings of the 8th European Workshop on Beam Diagnostics and Instrumentation for Particle Accelerators 2007 (JACoW, Venice, Italy, 2007), p. 180.

[13] C. Thomas, G. Rehm, I. Martin, and R. Bartolini, Phys. Rev. ST Accel. Beams 13, 022805 (2010).

[14] H. Sakai, M. Fujisawa, K. Iida, I. Ito, H. Kudo, N. Nakamura, K. Shinoe, T. Tanaka, H. Hayano, M. Kuriki, and T. Muto, Phys. Rev. ST Accel. Beams 10, 042801 (2007).

[15] S. Takano, M. Masaki, and H. Ohkuma, Nucl. Instrum. Methods Phys. Res., Sect. A 556, 357 (2006).

[16] T. Mitsuhashi, in Proceedings of the 5th European Workshop on Beam Diagnostics and Instrumentation for Particle Accelerators 2001 (JACoW, Grenoble, France, 2001), p. 26.

[17] M. Masaki and S. Takano, J. Synchrotron Radiat. 10, 295 (2003).

[18] F. Sannibale, G. V. Stupacov, M. S. Zolotorev, D. Filippetto, and L. Jägerhofer, Phys. Rev. ST Accel. Beams 12, 032801 (2009).
[19] Undulators, Wigglers and Their Applications, edited by H. Onuki and P. Elleaume (Taylor and Francis, London, 2003), Chap. 3, p. 69.

[20] O. Chubar, A. Snigirev, S. Kuznetsov, T. Weitkamp, and V. Kohn, in Proceedings of the 5th European Workshop on Beam Diagnostics and Instrumentation for Particle Accelerators 2001 (JACoW, Grenoble, France, 2001), p. 88.

[21] S. Takano, M. Masaki, A. Mochihashi, H. Ohkuma, M. Shoji, K. Tamura, H. Sumitomo, and M. Yoshioka, in Proceedings of the 1st International Beam Instrumentation Conference 2012 (JACoW, Tsukuba, Japan, 2012), p. 186.

[22] M. Masaki, S. Takano, K. Tamura, A. Mochihashi, M. Oishi, M. Shoji, T. Fujita, T. Takashima, H. Ohkuma, and S. Yamamoto, AIP Conf. Proc. 1234, 560 (2010).

[23] M. Masaki, A. Mochihashi, H. Ohkuma, S. Takano, and K. Tamura, in Proceedings of the 1st International Beam Instrumentation Conference 2012 (JACoW, Tsukuba, Japan, 2012), p. 492.

[24] F. Wang and S. Y. Lee, Phys. Rev. ST Accel. Beams 11, 050701 (2008).

[25] H. Tanaka, K. Kumagai, N. Kumagai, M. Masaki, H. Ohkuma, K. Soutome, and M. Takao, in Proceedings of the 7th European Particle Accelerator Conference, Vienna, Austria, 2000 (EPS, Geneva, 2000), p. 1575.

[26] G. Liu, K. Kumagai, N. Kumagai, H. Ohkuma, K. Soutome, M. Takao, and H. Tanaka, in Proceedings of the 18th Particle Accelerator Conference, New York, 1999 (IEEE, New York, 1999), p. 2337.

[27] J. Schimizu, K. Soutome, M. Takao, and H. Tanaka, in Proceedings of the 13th Symposium on Accelerator Science and Technology, edited by K. Sato, Osaka, Japan, (unpublished).

[28] S. Y. Lee, Accelerator Physics, 2nd ed. (World Scientific, Singapore, 2004), Chap. 4, p. 463. 